\title{
AGEING
}

\section{Old bone removal}

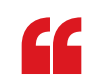

Moreover, injection of UBX0101 also produced a prochondrogenic environment that indicates new cartilage growth in young mice g
Senescent cells (SNCs), which accumulate in many tissues with age, contribute to several age-related pathologies. Now, Elisseeff and colleagues have shown that the selective elimination of SNCs with a specific inhibitor (UBX0101) of the anti-apoptotic protein mouse double minute 2 (MDM2) can prevent and even reverse the progression of osteoarthritis, an age-associated chronic disease that causes joint pain in $80 \%$ of people over 65 .

As senescent chondrocytes are found in cartilage tissue from patients undergoing joint replacement surgery, and this type of trauma is a risk factor for the development of osteoarthritis, the authors wondered whether SNCs contribute to the pathogenesis of osteoarthritis. To test this hypothesis, they analysed the presence of SNCs after anterior cruciate ligament transection (ACLT) in young p16-3MR mice, in which SNCs that express INK4A

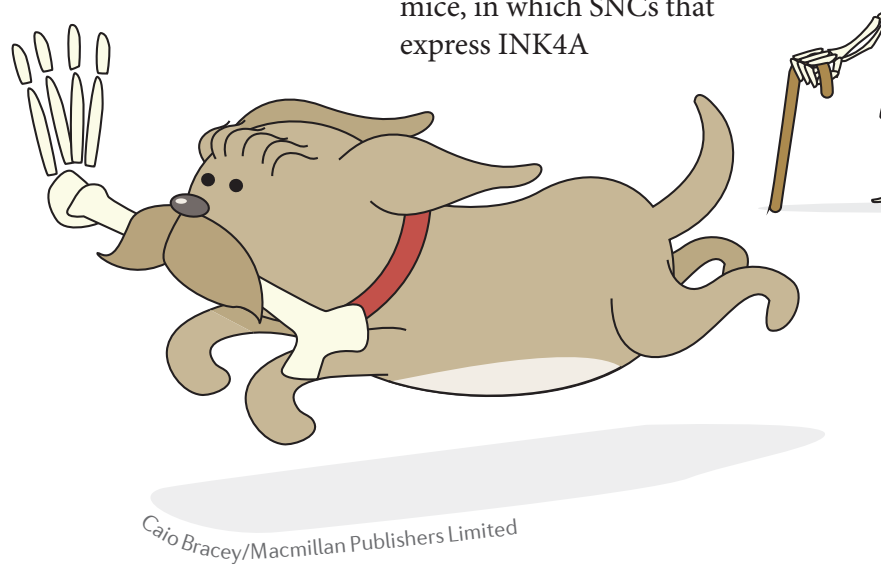

(also known as p16; a frequently used biomarker for SNCs) can be tracked by luminescence. ACLT increased the presence of SNCs in the articular joint region and synovium, and histologic and pain assessment confirmed development of osteoarthritis in these mice compared with sham-operated control mice. More severe osteoarthritis developed after ACLT surgery in aged mice than in young mice, which demonstrates the synergistic impact of age and trauma in osteoarthritis pathogenesis.

Next, Elisseeff and colleagues tested whether selective removal of SNCs with UBX0101 would reduce or reverse the progression of osteoarthritis and related symptoms.

UBX0101 injected intraarticularly 14 days after ACLT selectively killed SNCs, inhibited articular cartilage erosion, rescued proteoglycan staining of normal tissue, and decreased the pain associated with ACLT injury and the levels of expression of osteoarthritis inflammatory markers such as matrix metalloproteinase 13 (Mmp13) and interleukin-1 $\beta$ (Illb). Moreover, injection of UBX0101 also produced a prochondrogenic environment that indicates new cartilage growth in young mice, although this was not observed in their aged counterparts.

Last, the authors evaluated the clinical relevance of SNCs and treatment with UBX0101 in human articular cartilage samples explanted from patients with osteoarthritis who had undergone a total knee arthroplasty. UBX0101 cleared 20\% of senescence-associated $\beta$-galactosidase (SA- $\beta$-Gal)-positive SNCs and no more than $5 \%$ of non-SNCs. Moreover, treatment with UBX0101 increased the proliferation rate of non-senescent chondrocytes by up to $15 \%$. Exposure of human osteoarthritis chondrocytes to UBX0101 for 2 days showed a decline in expression of senescence and osteoarthritisrelated genes, such as $C D K N 2 A$, $M M P 13$ and $I L 1 B$, and increased the levels of $C O L 2 A 1$, proteoglycan and sulfated glycosaminoglycans (sGAGs), which confirms new cartilage growth.

These findings support elimination of SNCs as a promising approach for the treatment of both trauma and age-related degenerative joint diseases.

M. Teresa Villanueva

ORIGINAL ARTICLE Jeon, O. H. et al. Local clearance of senescent cells attenuates the development of post-traumatic osteoarthritis and creates a pro-regenerative environment. Nat. Med. 23, 775-781 (2017) 